\title{
Neuroprotective effects of Ellagic acid on Neonatal Hypoxic Brain Injury via Inhibition of Inflammatory Mediators and Down-regulation of JNK/p38 MAPK Activation
}

\author{
Si-Yan Chen ${ }^{1}$, Kuang Zheng ${ }^{2 \star}$ and Zhi-quan Wang ${ }^{3}$ \\ ${ }^{1}$ Department of Neurology, ${ }^{2}$ Department of Neurosurgery, The First Affiliated Hospital of Wen Zhou Medical College, Wenzhou,
} Zhejiang - 325000, China, ${ }^{3}$ Department of Biochemistry and Molecular Biology, Mayo Clinic, USA

*For correspondence: Email: zhengkuangzk@hotmail.com; Tel/Fax: 0086-577-55579351

Received: 28 September 2015

Revised accepted: 6 January 2016

\begin{abstract}
Purpose: To investigate if ellagic acid exerts neuroprotective effects in hypoxic-ischemic (HI) brain injury by inhibiting apoptosis and inflammatory responses.

Methods: Separate groups of rat pups from post-natal day 4 (D4) were administered with ellagic acid (10, 20 or $40 \mathrm{mg} / \mathrm{kg}$ body weight) orally till post-natal day 10 (D10). On D10, the rats were subjected to HI brain injury. Following HI injury, infarct size, weight and volume of the brain were measured. Apoptosis was assessed by Fluoro-Jade $C$ staining. Expression of caspases (caspase-3, 8 and 9), apoptotic pathway proteins (Bax, Bad, Bcl-2 and Bcl-xL), MAPKs, NF-KB(p65) and $p-I K-B \alpha$ were assessed by western blotting. mRNA levels of inflammatory mediators (TNF- $\alpha, I L-1 \alpha, I L-1 \beta$, iNOS, COX-2) were analyzed.

Results: Ellagic acidmarkedly $(p<0.05)$ reduced infarct size, volume and tissue loss. Significant $(p<$ $0.05)$ reduction in neuroapoptosis was observed on pre-treatment with ellagic acid. Expression levels of caspases, apoptotic pathway proteins and MAPK proteins were down-regulated with marked $(p<0.05)$ suppression of inflammatory mediators, $N F-K B(p 65)$ and $p-I K-B \alpha$.

Conclusion: Ellagic acid affords neuroprotection in HI brain injury by inhibiting apoptosis, inflammatory responses and modulating the proteins of apoptotic and MAPK pathways. Thus, ellagic acid may be a potent candidate for the treatment of HI injury.
\end{abstract}

Keywords: Brain injury, Ellagic acid, Hypoxia, Inflammatory mediators, Mitogen-activated protein kinases, Neuroprotective

Tropical Journal of Pharmaceutical Research is indexed by Science Citation Index (SciSearch), Scopus, International Pharmaceutical Abstract, Chemical Abstracts, Embase, Index Copernicus, EBSCO, African Index Medicus, JournalSeek, Journal Citation Reports/Science Edition, Directory of Open Access Journals (DOAJ), African Journal Online, Bioline International, Open-J-Gate and Pharmacy Abstracts

\section{INTRODUCTION}

Neonatal hypoxic-ischemic $(\mathrm{HI})$ brain injury is a major cause of perinatal mortality and morbidity [1]. Perinatal $\mathrm{HI}$ has an incidence rate of 18 cases in every 1000 births, affecting approximately $60 \%$ of pre-term infants [2]. HI brain injury arising in neonatal and perinatal stages damages brain cell development, resulting in long-lasting neurologic sequelae with cerebral palsy, epilepsy, learning disabilities and motor disability as complications [3]. Brain injury in perinatal stage has multifaceted aetiology involving inflammation, a major cause in the progression of $\mathrm{HI}$-induced injury in neonates and in adults [4]. Inflammation is involved in sensitizing the neonatal brain to ischaemia 
through pro-inflammatory cytokines which are found in increased levels [5].

Mitogen activated protein kinases (MAPKs) p38s, extracellular signal-regulated kinases (ERKs) and c-Jun $\mathrm{NH}_{2}$-terminal kinases (JNKs) are activated in mammalian cells in response to various growth factors, cytokines and agents that cause cell damage [6]. The activated MAPKs principally function as mediators of cellular stress by phosphorylating various transcription factors, cytosolic proteins and intracellular enzymes that are chiefly involved in cell survival, apoptosis and in expression of inflammatory mediators [7].

Recently much of research work is focusing on plant-derived compounds for their neuroprotective effects. Ellagic acid is found in numerous plants, predominantly in fruits and nuts such as cranberries, pomegranates, raspberry, strawberries and walnuts [8]. Previous researchers have reported that ellagic acid possesses various pharmacological properties through regulation of several pathways such as glycogen synthase kinase-3 beta (GSK-3 $\beta$ )/ phosphoinositide 3-kinase (PI3-K) signaling [9]; regulation of cytokines [10]; activating antioxidant response [11]; and modulating the cell-cycle/cell survival genes [12]. Considering these biological effects the present study aimed to explore the influence of ellagic acid in neonatal $\mathrm{HI}$ brain injury.

\section{EXPERIMENTAL}

\section{Chemicals and reagents}

Ellagic acid was purchased from Sigma-Aldrich, St.Louis, MO, USA. Antibodies against caspase3, caspase-8, caspase-9, Bcl-xL, Bcl-2, Bax, Bad (Cell Signalling Technology, Danvers, MA, USA). $\beta$-actin, JNK, phospho-JNK, ERK1/2, phosphoERK1/2, p38, phospho-p38, NF-kB(p65), p-IKBa (Santa Cruz Biotechnology, Santa Cruz, CA, USA) were used in expression analysis. All other chemicals and reagents were obtained from Sigma-Aldrich, St.Louis, MO, USA, unless otherwise specified.

\section{Experimental animals}

Pregnant Sprague Dawley rats (Guangdong Medical Laboratory Animal Co., China) used in this study was maintained on $12 \mathrm{~h}$ light/dark cycle with room temperature at $22 \pm 1^{\circ} \mathrm{C}$. The rats had ad libitum access to water and feed and were housed individually in separate cages and monitored closely for the day of litter of pups. The day of litter was noted as postnatal day 0
(Day0). The pups were kept in cages with their littermates in conditions similar to their dams. From day 4, separate groups of pups were administered with ellagic acid $(10,20$ or 40 $\mathrm{mg} / \mathrm{kg}$ b.wt, orally). On D10, one hour after treatment with ellagic acid, the pups were experimentally subjected to HI. Pups not treated with ellagic acid and not subjected to $\mathrm{HI}$ served as control, while pups exposed to $\mathrm{HI}$ injury and not treated with ellagic acid served as the $\mathrm{HI}$ control. For animal studies, ethical approval was obtained from the ethical committee of Wen Zhou Medical college (Approval Reference Number 4208211) and was performed in compliance with the guide for care and use of laboratory animals [13].

\section{Hypoxic-ischemic $(\mathrm{HI})$ brain injury procedure}

Ten-day-old pups were subjected to $\mathrm{HI}$ brain injury as described by Shen et al [14]. The left common carotid artery was ligated under anesthesia with $3 \%$ isoflurane. Following recovery period of $90 \mathrm{~min}$, the pups were placed in a humidified chamber containing atmosphere of $8 \% \mathrm{O}_{2} / 92 \% \mathrm{~N}_{2}$ for a period of $2.5 \mathrm{~h}$ and were then returned to their respective cages.

\section{Measurement of brain infarction}

Following $24 \mathrm{~h}$ of $\mathrm{HI}$ injury, the rat pups were sacrificed by decapitation and brain tissues were immediately dissected out and $1 \mathrm{~mm}$ coronal slices were immersed in a PBS solution containing $2 \%$ triphenyltetrazoliumchloride (TTC) at $37^{\circ} \mathrm{C}$ for $25 \mathrm{~min}$. The live/dead cells were determined based on the uptake of TTC. Live cells take up TTC where it is converted to red colour in live mitochondria. The viable tissues appear brick-red, and non-viable/infarcted tissue can be identified by the absence of red colour (white). The tissues were further analysed for infarct size and volume using $\mathrm{NIH}$ Image $\mathrm{J}$ imaging applications as described by Williams et al [15].

\section{Histological analysis by cresyl violet staining}

Histologic studies were performed $48 \mathrm{~h}$ after $\mathrm{HI}$ insult. The brain tissues were fixed in $4 \%$ paraformaldehyde and embedded in paraffin. Sections $(4-5 \mu \mathrm{m})$ were mounted on glass cover slides and were deparaffinized and rinsed with distilled water and incubated for $15 \mathrm{~min}$ in $1 \%$ cresyl violet solution. After incubation, the sections were rinsed in water and destained with $1 \%$ acetic acid solution and finally dehydrated by rinsing with ethanol. The slides were rinsed thoroughly and observed under microscopy, photographed and analysed [16]. 


\section{Evaluation of neuro-apoptosis}

Neuro-apoptosis was evaluated by immunohistochemical staining for activated caspase-3 and Fluoro-Jade $C$ staining. The analysis was performed as described previously [17]. The brain tissue sections were embedded in paraffin $(5 \mu \mathrm{m})$ and incubated overnight at $4{ }^{\circ} \mathrm{C}$ with anticleaved caspase-3 primary antibody (1:200; monoclonal antibody, Cell Signalling Technology, Beverly, MA, USA). The tissue sections were further incubated with secondary antibody (1:200, Santa Cruz Biotechnology, Inc., Santa Cruz, CA, USA) for $40 \mathrm{~min}$. The sections were then incubated with avidin-biotinylated peroxidase complex (Vectostain ABC-Kit, Vector Lab, Burlingame, CA, USA) for $40 \mathrm{~min}$ and stained with diaminobenzidine (DAB, Vector Laboratories, Burlingame, CA, USA). The immune-positive cells in the various sections of the brain tissue - hippocampal CA1, CA3 and dentate gyrus (DG) were analyzed using NISElements BR imaging processing and analysis software (Nikon Corporation, Japan).

For fluoro-Jade $\mathrm{C}$ staining, the tissue sections of $60 \mu \mathrm{m}$ thickness were stained with Fluoro-Jade $\mathrm{C}$, a marker very specific for neurodegeneration. The apoptotic cell counts were recorded as Fluoro-Jade positive cells using Nikon Eclipse 80i microscope under 20 x magnification.

\section{Western blot analysis}

The cytosolic and nuclear fractions were prepared and subjected to western blotting [18]. The samples were homogenized in lysis buffer(5 $\mathrm{M} \mathrm{NaCl},, 10 \%$ Nonidet P-40, $1 \mathrm{M}$ HEPES, $0.1 \mathrm{M}$ EGTA, $0.5 \mathrm{M}$ EDTA, $0.1 \mathrm{M}$ phenylmethylsulfonyl fluoride, $1 \mathrm{M}$ sodium fluoride, $0.2 \mathrm{M}$ sodium orthovanadate, $2 \mu \mathrm{g} / \mathrm{mL}$ aprotinin, and 2 $\mu \mathrm{g} / \mathrm{mL}$ leupeptin) and centrifuged at $20,000 \mathrm{~g}$ for $15 \mathrm{~min}$ at $4^{\circ} \mathrm{C}$. The supernatant was collected and protein content was determined using Beyotime protein assay kit (Beyotime Institute of Biotechnology, Nanjing, China). Equal amounts of protein $(40 \mu \mathrm{g})$ were separated by electrophoresis on sodium dodecyl sulfatepolyacrylamide gel electrophoresis gels (10\%) and then transferred to polyvinylidene fluoride membrane (Millipore, Billerica, USA). The membrane was blocked with $5 \%$ non-fat dry milk in tris-buffered saline containing $0.05 \%$ Tween20 (TBST) buffer and incubated overnight at $4{ }^{\circ} \mathrm{C}$ with primary antibodies. Membranes were washed thrice with TBST buffer and further incubated with secondary antibodies coupled to horseradish peroxidase (1:1000 dilution, Santa Cruz Biotechnology) for $2 \mathrm{~h}$ at room temperature. The immunoreactive bands were detected by enhanced chemiluminescence system according to the manufacturer's protocol (Millipore, Billerica, USA). The signals were analysed by scanning densitometry (Quantity One Software Bio-Rad Laboratories, Hercules, CA, USA). The expressed protein levels were normalized to that of $\beta$-actin.

\section{RT-PCR analysis}

Expression of the genes coding for inflammatory mediators in the cortex at $24 \mathrm{~h}$ following $\mathrm{H} / \mathrm{I}$ induction was analyzed. The mRNA levels of TNF- $\alpha$, iNOS, COX-2, IL- $1 \alpha$, IL- $1 \beta$ and IL- 6 and were determined. Total RNA was isolated from lysed cells using the RNeasy kit according to the manufacturer's instructions (Qiagen, Valencia, CA). RT-PCR was performed as described by Shen et al. [14]. Total RNA was amplified by PCR using the following primers- iNOS forwardGTGCTAATGCGGAAGGTCATG, reverseGCTTCCGACTTTCCTGTCTCAGTA; COX-2forward-TGTCCCTTTGCCTCTTTCAAT, reverse-GAGGCACTTGCGTTGATGGT; IL-1 $\alpha$ forward-AAGACAAGCCTGTGTTGCTGAAGG and TCCCAGAAGAAAATGAGGTCGGTC; IL$1 \beta$-forward -CACCTCTCAAGCAGAGCACAG, reverse- GGGTTCCATGGTGAAGTCAAC; IL-6forward- TCCTACCCCAACTTCCAATGCTC, reverse- TTGGATGGTCTTGGTCCTTAGCC; TNF- $\alpha$-forward-AAATGGGCTCCCTCTCATCAG TTC and, reverse- TCTGCTTGGTGGTTTGCTA CGAC and GAPDH forward -CCAGCCTCGT CTCATAGACA and reverse-GTAACCAGGCGT CCGATACG.

\section{Statistical analysis}

The results are presented as mean $\pm S D(n=3$ or 6). Data were analysed by one-way analysis of variance (ANOVA) followed by post-hoc analysis using Duncan's Multiple Range Test. The data obtained were analysed using SPSS software (version 22.0).Differences were considered statistically significant at $p<0.05$.

\section{RESULTS}

\section{Ellagic acid pretreatment reduced brain infarct}

Extensive infarction was seen in the cerebral cortical and sub-cortical areas in rats subjected to $\mathrm{HI}$ (Fig. 1). Rats pre-treated with ellagic acid $(10,20$ or $40 \mathrm{mg} / \mathrm{kg})$ had significantly $(p<0.05)$ reduced infarct volumes in comparison to $\mathrm{HI}$ control. Further a striking rise in infract weight was observed in $\mathrm{HI}$ induced rats pups. Ellagic acid treatment remarkably reduced the infarct 
weight. $40 \mathrm{mg}$ dose brought more significant reductions than lower doses, suggesting dosedependent reduction in infarct weight and volume.

\section{Effect of ellagic acid on brain tissue histology following $\mathrm{HI}$}

Cresyl violet staining revealed the morphology of normal viable cells as round and pale stained nuclei throughout the tissue sections of cerebral cortex, hippocampus and striatum (Fig. 2). Higher numbers of cells under apoptosis were observed as shrunken cells with pyknotic nuclei. Further, large areas of tissue loss were also observed in the ipsilateral cerebral cortex, striatum and hippocampus of $\mathrm{HI}$ control animals. Ellagic acid treatment decreased the apoptotic cell counts and also reduced the extent of tissue damage. Hippocampus had more injury when compared to the cortex and striatum. While 10 $\mathrm{mg}$ ellagic acid did reduce the degree of brain injury, 20 and $40 \mathrm{mg}$ doses were more effective. Ellagic acid $(40 \mathrm{mg})$ reduced the tissue loss to nearly $13.1 \%$ in the hippocampus as against $78.51 \%$ in pups that are not treated.

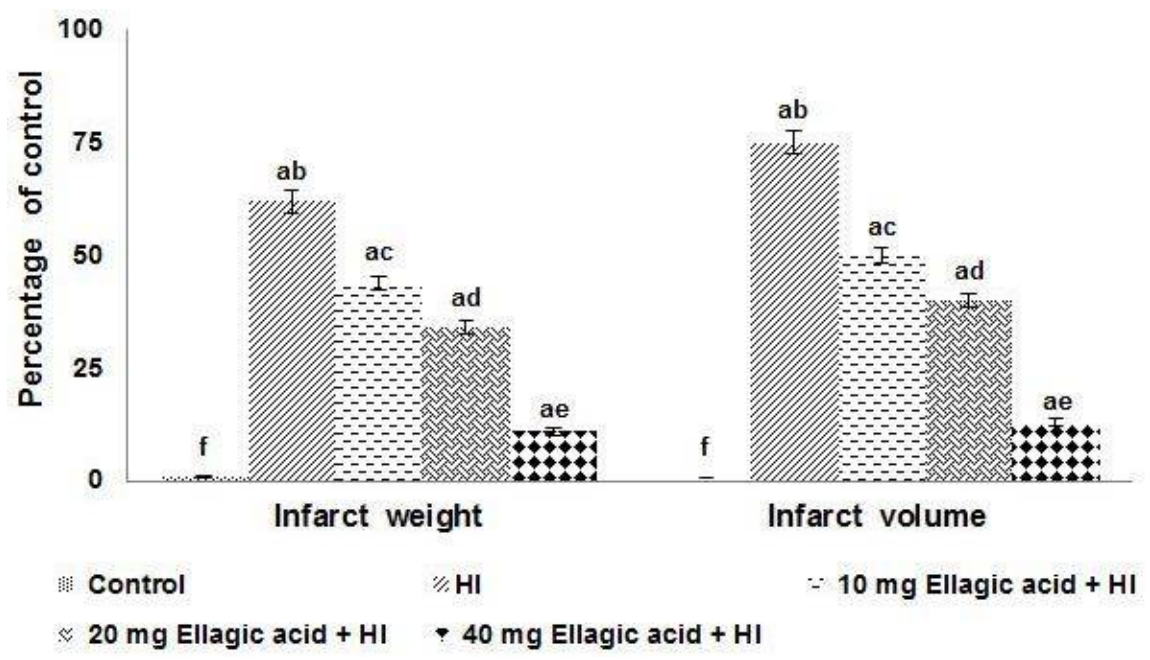

Figure 1: Effect of Ellagic acid on infarct weight and volume following $\mathrm{HI}$. The results are represented as mean \pm $\mathrm{SD}(\mathrm{n}=6)$;' $\mathrm{a}$ ' represents statistical significance at $p<0.05$ compared to control; and 'b' to ' $\mathrm{f}$ ' represent significant difference $(p<0.05)$ between mean values of different groups as determined by one-way ANOVA followed by DMRT analysis

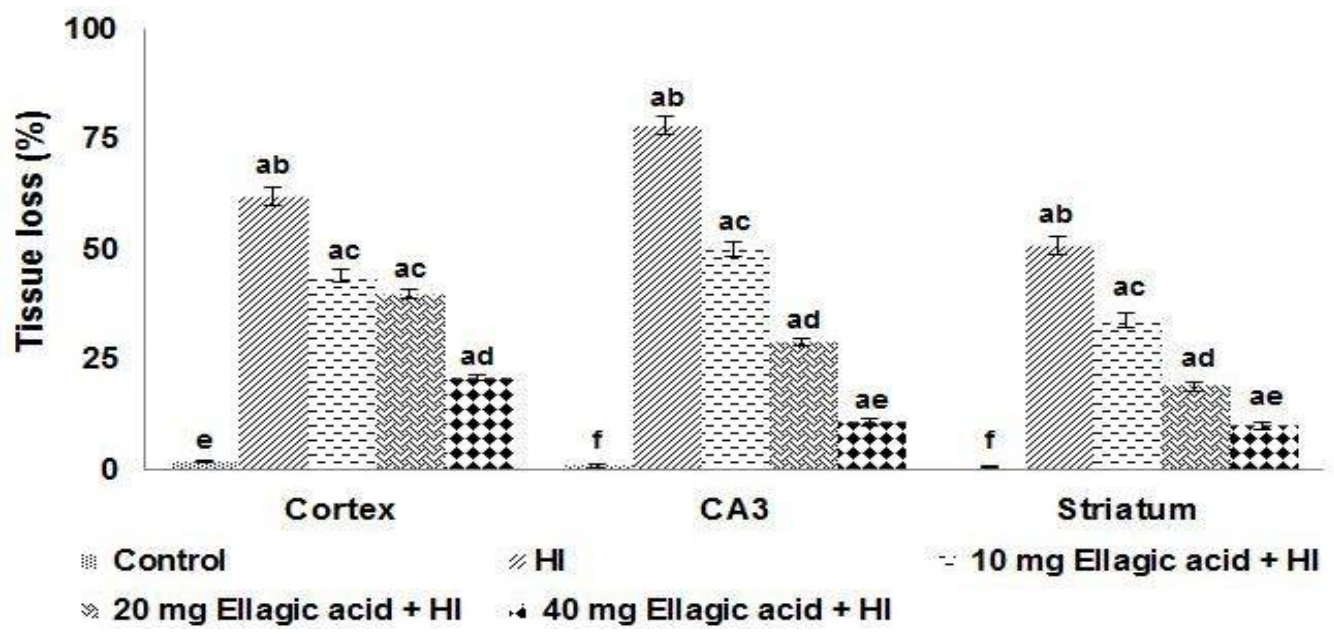

Figure 2: Effect of Ellagic acid on the intensive tissue loss following HI. Significant $(p<0.05)$ reduction in brainloss following ellagic acid treatment was observed post-HI-injury with maximum protection at $40 \mathrm{mg}$ dose. Values are represented as mean $\pm \mathrm{SD}, \mathrm{n}=6$. A represents statistical significance at $p<0.05$ compared against control and b-f represents significant difference $(p<0.05)$ between mean values of different experimental groups as determined by one-way ANOVA followed by DMRT analysis 
Ellagic acid markedly inhibits neuronal apoptosis following HI-induced brain injury

Activation of caspase- 3 was considered as a measure of apoptosis. Caspase- 3 positive cells were detected in CA1 and CA3 areas of hippocampus and in the DG. $\mathrm{HI}$ resulted in a striking $(p<0.05)$ increase in number of caspase-3 positive cell counts (Fig. 3). The expression levels of caspase- 3 as determined by western blot analysis indicated significant increase $(p<0.05)$ following HI-injury (Fig 4). Ellagic acid $(10,20$ or $40 \mathrm{mg})$ pre-treatment significantly $(p<0.05)$ inhibited caspase-3 activation as compared to $\mathrm{HI}$ control pups. Immuno-reactivity towards cleaved caspase- 3 in the brain tissues exhibited a dramatically reduced number of caspase-3-positive cells in the hippocampus of animals administered ellagic acid. The decrease in the expression of caspase3 and in the number of apoptotic cell counts was observed to be dose-dependent. Furthermore, increased expression level of caspases- 8 and 9 in $\mathrm{HI}$ was significantly reduced on ellagic acid treatment (Fig. 4). Also the Fluoro-Jade C positive cells $(p<0.03)$ were found to be lowered on ellagic acid pre-treatment (Fig. 3).

\section{A}

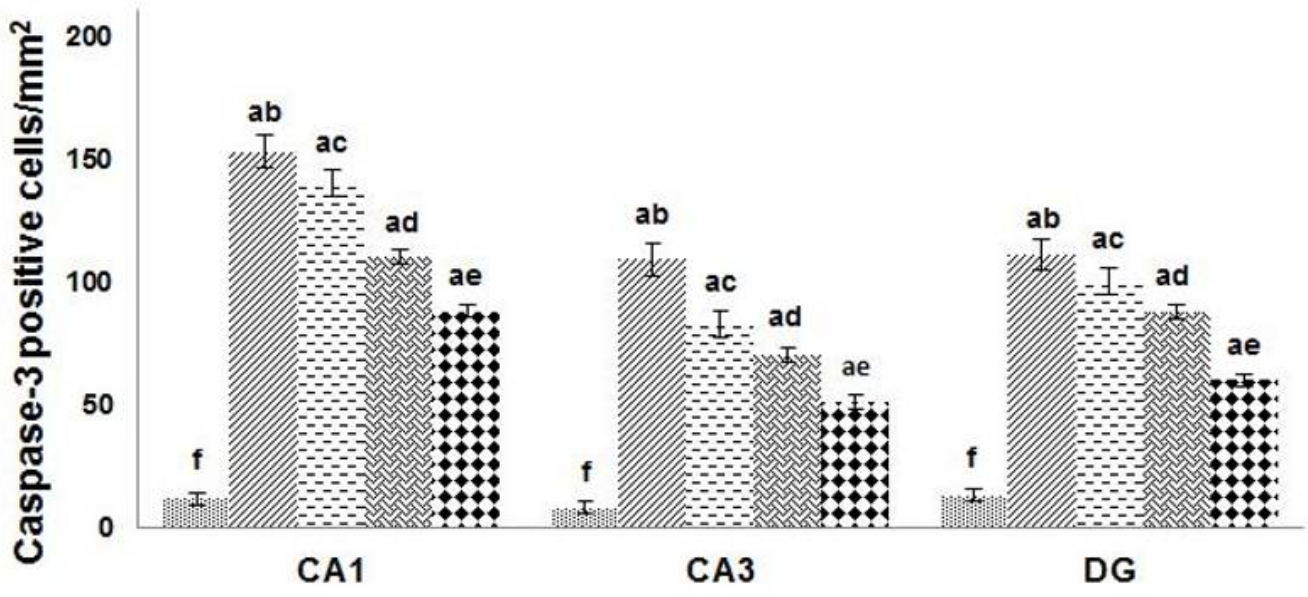

17. Control $\% \mathrm{H}$

$\therefore 10 \mathrm{mg}$ Ellagic acid $+\mathrm{HI}$

$20 \mathrm{mg}$ Ellagic acid + HI $: 40 \mathrm{mg}$ Ellagic acid + H

B

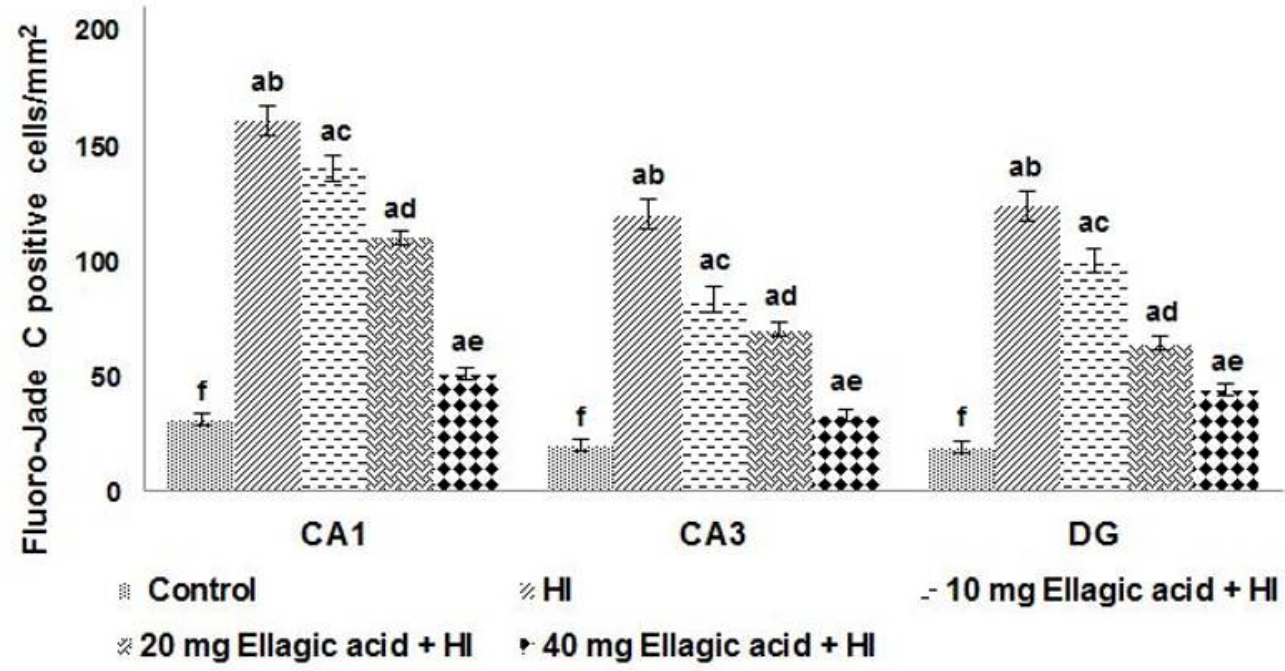

Figure 3: Effect of Ellagic acid on the intensive apoptotic neurodegeneration due to HI. Ellagic acid caused significant decrease in the expressions of activated caspase- 3 expressions (A) and apoptotic cell counts (B)Values are represented as mean $\pm S D, n=6$. a represents statistical significance at $p<0.05$ compared against control and $b$-f represents significant difference $(p<0.05)$ between mean values as determined by one-way ANOVA followed by DMRT analysis 


\section{Effect of ellagic acid on apoptotic pathway proteins}

The expression of pro-apoptotic (Bad and Bax) and anti-apoptotic proteins (Bcl-2 and $\mathrm{Bcl}-\mathrm{xL}$ ) were assessed following $\mathrm{HI}$ insult. Bcl-2 family proteins are important regulators of apoptosis. In our study, $\mathrm{HI}$-insult resulted in multi-fold increase in expression of $\mathrm{Bad}$ and Bax proteins with significant decrease $(p<0.05)$ in $B c l-2$ and $B c l-x L$ expressions. Ellagic acid administration caused a tremendous decline in the expression levels of pro-apoptotic proteins while it enhanced survival proteins- Bcl-xL and Bcl-2 (Fig. 4). The expression of proteins were in line with the observed caspase-3 expression and apoptotic cell counts.

A

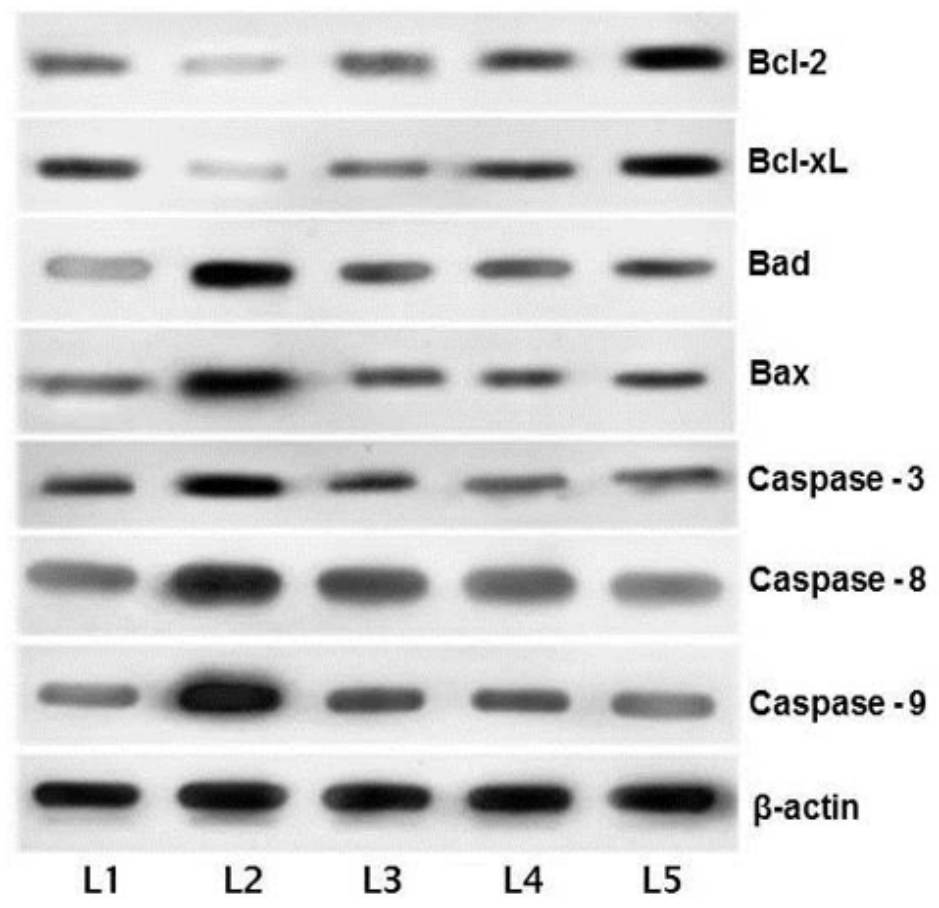

B

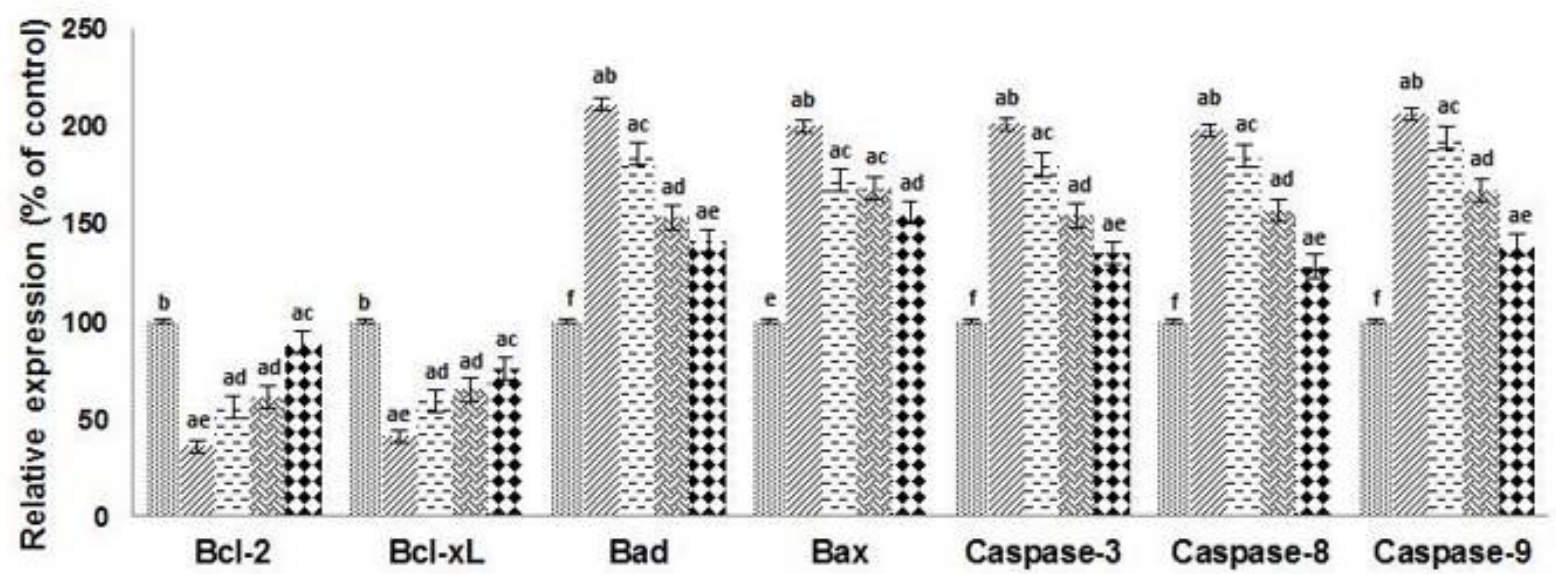

ai Control $\% \mathrm{HI} \quad=10 \mathrm{mg}$ Ellagic acid $+\mathrm{HI} \approx 20 \mathrm{mg}$ Ellagic acid $+\mathrm{HI} \quad 240 \mathrm{mg}$ Ellagic acid $+\mathrm{HI}$

Figure 4: Effect of Ellagic acid on the expressions of apoptotic pathway proteins. Ellagic acid markedly downregulated the expressions of caspases, Bad and Bax while elevating the expressions of $B c l-2$ and $B c l-x L(A)(L 1-$ Control; L2-HI; L3-10 mg Ellagic acid +HI; L4-20 mg Ellagic acid +HI; L5-40 mg Ellagic acid +HI); (B) Relative expressions. Values are represented as mean $\pm \mathrm{SD}, \mathrm{n}=6$. a represents statistical significance at $p<0.05$ compared against control and b-f represents significant difference $(p<0.05)$ between mean values as determined by one-way ANOVA followed by DMRT analysis 


\section{Ellagic acid modulated the expression of MAPK proteins}

The results reveal a significantly $(p<0.05)$ raised levels of JNK, $p-J N K, p-38$ and $p-p-38$. Not much rise in ERK $1 / 2$ expressions were observed. Ellagic acid strikingly down-regulated the expressions of JNK and p38MAPK (Fig. 5). While significant suppression of JNK was observed, ellagic acid brought about only a small decrease in the expression of ERK1/2. The inhibitory effects of ellagic acid observed were dosedependent.

\section{Influence of ellagic acid on expression of inflammatory mediators}

A striking increase in the expression of p65 NF$\mathrm{KB}$ and $\mathrm{p}-\mathrm{IK}-\mathrm{Ba}$ on $\mathrm{HI}$-injury was observed. Interestingly, ellagic acid administration caused a remarkable decrease in NF-kB and p-IK-Ba (Fig. 6 ) and also in the mRNA levels of TNF- $\alpha$, COX2, IL-1 $\alpha, I L-6, I L-1 \beta$ and iNOS (Fig 7).

A

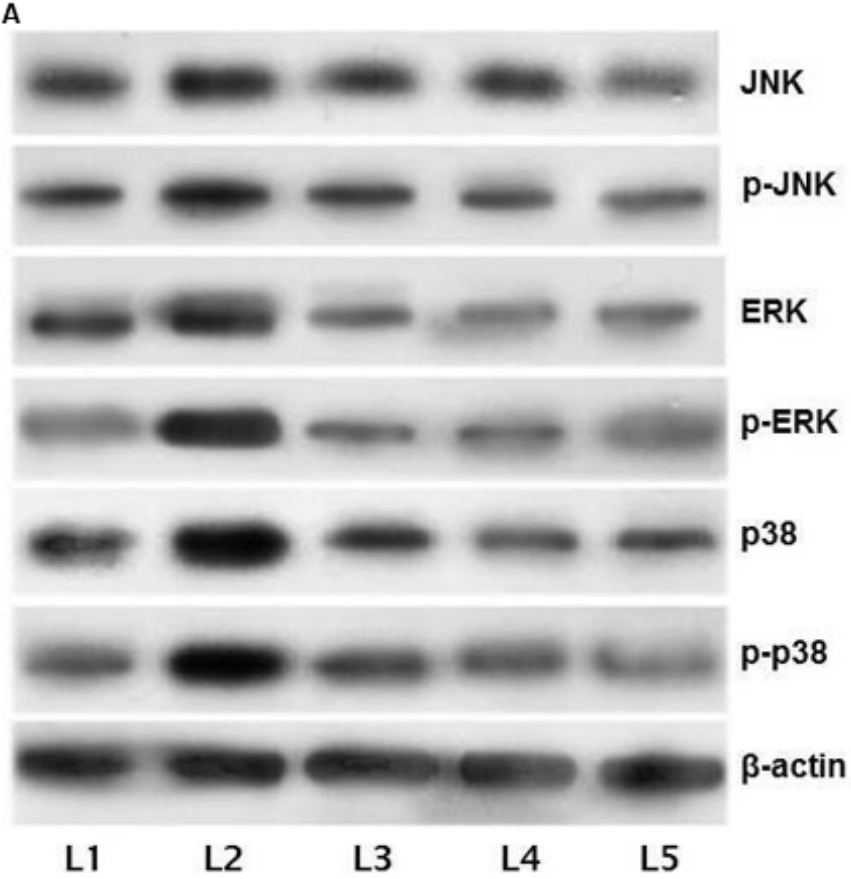

B

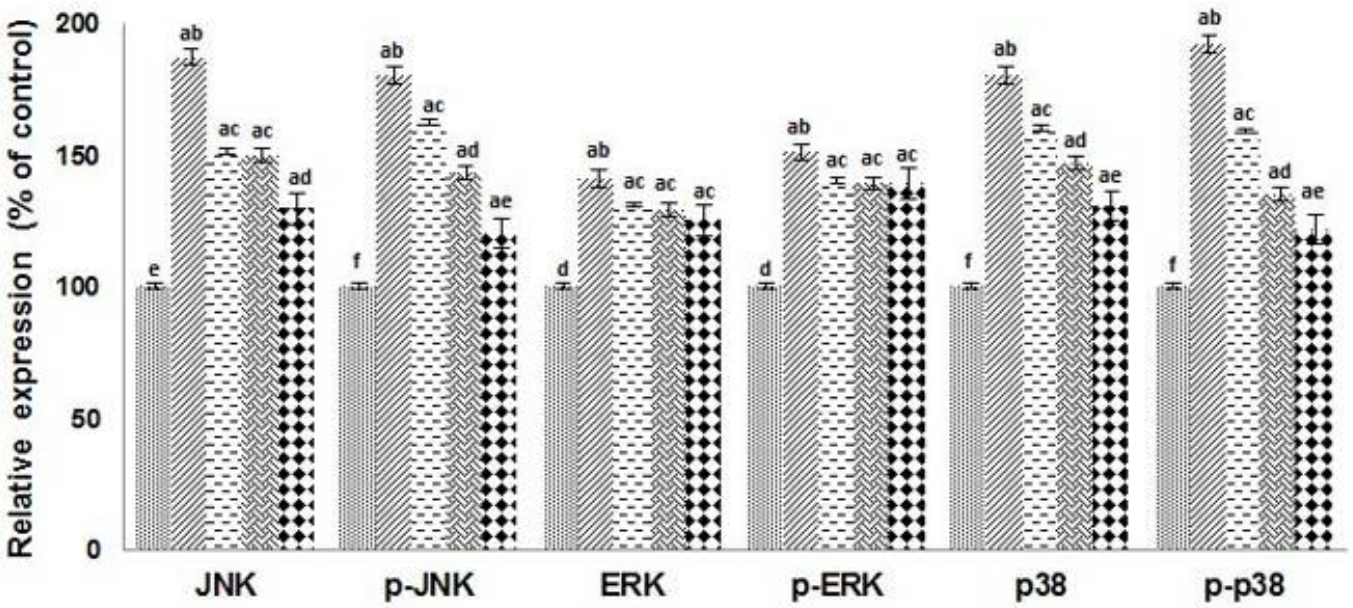

Control $\because \mathrm{HI} \because 10 \mathrm{mg}$ Ellagic acid $+\mathrm{H} \geqslant 20 \mathrm{mg}$ Ellagic acid $+\mathrm{HI} * 40 \mathrm{mg}$ Ellagic acid $+\mathrm{HI}$

Figure 5: Effect of Ellagic acid on the expressions of MAPK pathway proteins. (A) L1-Control; L2-HI; L3-10 mg Ellagic acid $+\mathrm{HI}$; L4-20 mg Ellagic acid $+\mathrm{HI}$; L5-40 mg Ellagic acid $+\mathrm{HI}(\mathrm{B})$. Values are represented as mean \pm $\mathrm{SD}, \mathrm{n}=6$. a represents statistical significance at $p<0.05$ compared against control and b-f represents significant difference $(p<0.05)$ between mean values as determined by one-way ANOVA followed by DMRT analysis 
A

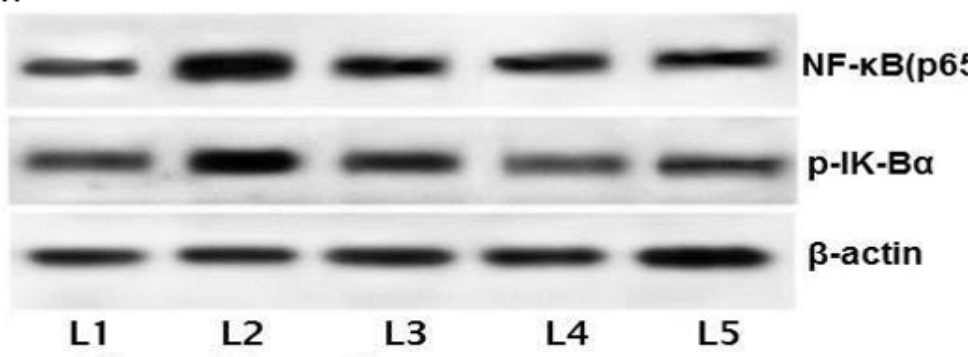

B

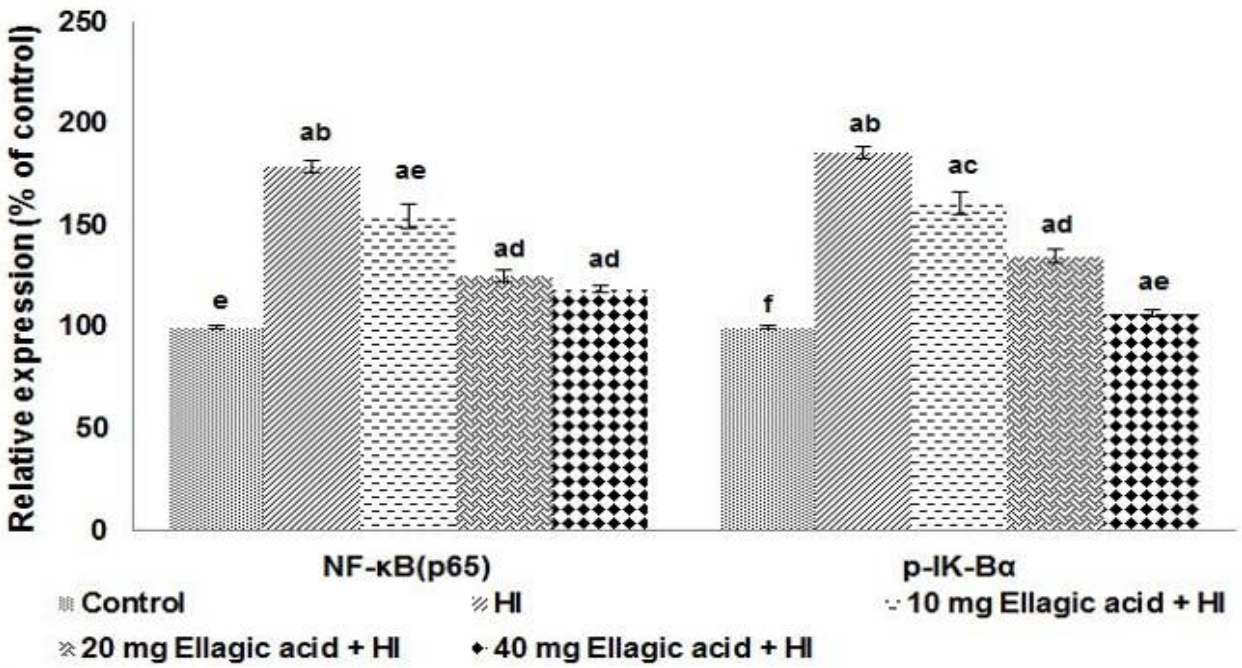

Figure 6: Effect of Ellagic acid on the expressions NF-KB and IK-Ba following HI-injury. Ellagic acid pre-treatment caused striking decreases in the activated forms of NF-KB and IK-Ba (A). L1-Control; L2-HI; L3-10 mg Ellagic acid + HI; L4-20 mg Ellagic acid +HI; L5-40 mg Ellagic acid $+\mathrm{HI}(\mathrm{B})$. Values are represented as mean \pm SD, $n=6$. a represents statistical significance at $p<0.05$ compared against control and $b$-f represents significant difference $(p<0.05)$ between mean values as determined by one-way ANOVA followed by DMRT analysis

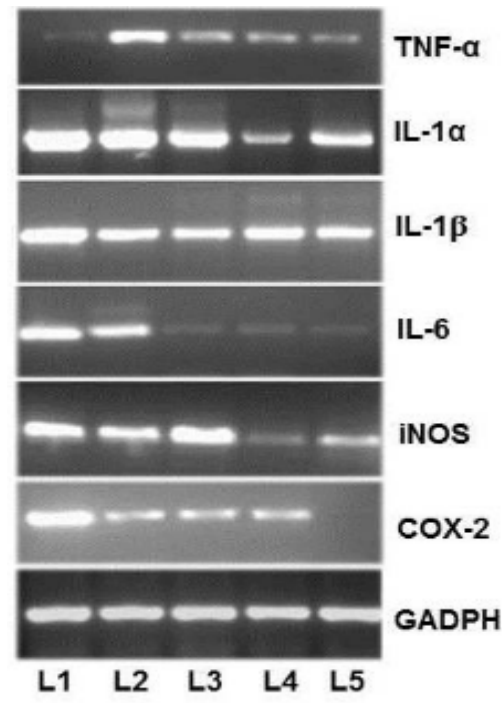

Figure 7: Effect of ellagic acid on the expressions inflammatory mediators following HI-injury. (L1Control; L2-HI; L3-10 mg ellagic acid +HI; L4-20 mg ellagic acid $+\mathrm{HI}$; L5-40 mg ellagic acid $+\mathrm{HI}$ ). Ellagic acid potentially inhibited the expressions of inflammatory mediators post-HI-injury. Significantly ( $p$ $<0.05$ ) enhanced expressions of the inflammatory mediators following $\mathrm{HI}$ injury were effectively downregulated by Ellagic acid in a dose-dependent manner.

\section{DISCUSSION}

Perinatal $\mathrm{HI}$ brain injury is a common cause of mortality and neurological deficits in infants and children [19]. The pathological process that may possibly contribute to neuronal death and dysfunction includes inflammatory responses, apoptosis, excitotoxicity and oxidative stress [20]. Unfortunately, the therapeutic interventions are limited.

In this study, ellagic acid (10, 20 or $40 \mathrm{mg} / \mathrm{kg}$ ) administered for a week significantly protected the neonatal brain from $\mathrm{HI}$ injury. The infarct size and volume were reduced. Studies have reported that neonatal brain cells exhibit features of apoptosis following $\mathrm{HI}$ insult [21].Apoptosis has been reported as a typical mechanism of cell death in the developing brain. Cresyl violet and Fluoro-Jade C staining revealed enhanced apoptosis that was significantly reduced by ellagic acid. Further, it has been reported that both caspase-dependent [22] and caspaseindependent [23] pathways are activated in brain injury after HI. Caspase-3 activation is considered as a reliable marker of the apoptotic neuronal cell death in $\mathrm{HI}$ brain injury [24]. 
Western blot analysis revealed enhanced expression of caspases- 3,8 and 9 in the brain tissues of neonatal rats following $\mathrm{HI}$. The activation of caspases 8 and 9 eventually leads to the activation of caspase-3 [25]. Thus increases in caspase- 8 and 9 expression reflects the expression of caspase-3.The increase was in line with the apoptotic cell counts observed, indicating the activation of the caspase cascades that were effectively down-regulated by ellagic acid.

Neuro-inflammation is implicated in hypoxic injuries of neonatal brain [26]. Inflammatory cytokines- TNF- $\alpha$ and IL-1 $\beta$, along with nitric oxide (NO) produced by activated microglial cells lead to neuronal injury [27].Also targeting MAPK signalling pathways in cerebral injury is valuable in treatment as these pathways are associated with apoptotic signalling as well [28]. Studies have reported that inflammatory mediators produced in cerebral $\mathrm{HI}$ injury activate MAPK signalling cascades [29,30]. Our results demonstrate significant activation of JNK, ERK $1 / 2$ and p38 suggesting activation of MAPK cascades. Strikingly reduced levels of JNK, $p$ JNK, p38and p-38 were observed on ellagic acid pre-treatment, only negligible inhibitory effects were noticed on ERK1/2 and p-ERK1/2. Earlier researches have demonstrated that activated JNK and p38 MAPKs chiefly function to arbitrate cellular stress in cerebral ischemic injury by regulating the activities of intracellular enzymes and transcription factors, associated with cell survival and apoptosis [29]. Thus, suppression of JNK and p-38 MAPK and inflammatory mediators could have possibly contributed to the decrease in neuronal apoptosis.

$\mathrm{HI}$ injury resulted in a drastic increase in the expression of NF-KB p65 and $\mathrm{p}-\mathrm{I} \mathrm{KB} \alpha$. NF-KB regulates genes involved in inflammation and stress responses [31]. Under pathological conditions, IKB is phosphorylated resulting in active NF-KB [32] and gets translocated to the nucleus where it regulates target gene expression. An increase in phosphorylated NF$\mathrm{KB}$ in hypoxic microglia of neonatal rats has been reported previously [33]. Significant increase in the expression levels of IL-6, COX-2, NF-KB and iNOS were also observed. Marked suppression of NF-kB p65, IL -1 $\beta, \mathrm{IL}-1 \alpha$ and $p$-I $\mathrm{kB} \alpha$ suggests the potent anti-inflammatory efficiency of ellagic acid. Enhanced p65NF-kB expression observed was in line with the expression of TNF- $\alpha$, COX-2, IL-1 $\alpha$, IL-1 $1 \beta$, IL- 6 and iNOS following HI. Recent studies reported that the functions of TNF- $\alpha$ and IL-1 $\beta$ were mediated through caspase cascades and iNOS [34]. The down-regulation of inflammatory mediators thus curbs inflammation, suggesting anti-inflammatory effects as one of the mechanisms involved in neuroprotection by ellagic acid.

\section{CONCLUSION}

Ellagic acid reduces brain infarct size and apoptosis of brain cells through mechanisms such as down-regulation of apoptotic pathway proteins, suppression of MAPK cascades and expression of inflammatory mediators. MAPK cascades are critically associated with cell survival and apoptosis. Furthermore, neuroinflammation adsorbed in $\mathrm{HI}$ potentially contributes to apoptosis. Down-regulation of MAPKs and inflammatory protein expression by ellagic acid reveals its protective effects in $\mathrm{HI}$. The results suggest that ellagic acid may be useful in therapeutic strategies for brain injury. However, the potential neuroprotective effects of ellagic acid require further investigation.

\section{ACKNOWLEDGEMENT}

The author would like to express their gratitude to $\mathrm{Dr}$ Wei-Dong Chen for his advice on experiments.

\section{REFERENCES}

1. Graham EM, Ruis $K A$, Hartman AL, Northington FJ, Fox HE. A systematic review of the role of intrapartum hypoxia-ischemia in the causation of neonatal encephalopathy. Am J Obstet Gynecol 2008; 199: 587595.

2. Ferrieo DM. Neonatal brain injury. N Engl J Med 2004; 35: 1985-1995.

3. Lorenz JM, Wooliever DE, Jetton JR, Paneth N. A quantitative review of mortality and developmental disability in extremely premature newborns. Arch Pediatr Adolesc Med 1998; 152: 425-435.

4. Calvert JW, Zhang JH. Pathophysiology of an hypoxicischemic insult during the perinatal period. Neurol Res 2005; 27: 246-260.

5. Wang $X$, Stridh L, Li W, Dean J, Elmgren A, Gan L, Eriksson K, Hagberg H, Mallard C. Lipopolysaccharide sensitizes neonatal hypoxic-ischemic brain injury in a MyD88-dependent manner. J Immunol 2009; 183: 74717477.

6. Cuenda A, Rousseau S. p38 MAP-Kinases pathway regulation, function and role in human diseases. Biochimica et Biophysica Acta 2007; 1773: 1358-1375.

7. Kyriakis JM, Avruch J. Mammalian MAPK signal transduction pathways activated by stress and inflammation: a 10-year update. Physiol Rev 2012; 92: 689-737.

8. Whitley AC, Stoner GD, Darby MV, Walle T. Intestinal epithelial cell accumulation of the cancer preventive

Trop J Pharm Res, February 2016; 15(2): 249 
polyphenol ellagic acid extensive binding to protein and DNA. Biochem Pharmacol 2003; 66: 907-915.

9. Kowshik J, Giri H, Kishore TK, Kesavan R, Vankudavath RN, Reddy GB, Dixit $M$, Nagini S. Ellagic acid inhibits VEGF/VEGFR2, PIJK/Akt and MAPK signaling cascades in the hamster cheek pouch carcinogenesis model. Anticancer Agents Med Chem 2014; 14: 12491260.

10. Favarin DC, Teixeira $M M$, de Andrade $L M$, Alves $C F$, Chica JML, Sorgi CA, Faccioli LH, Rogerio AP. Antiinflammatory effects of ellagic acid on acute lung injury induced by acid in mice. Mediators Inflamm 2013; 164202.

11. Hseu YC, Chou CW, SenthilKumar KJ, Fu KT, Wang HM, Hsu LS. Ellagic acid protects human keratinocyte (HaCaT) cells against UVA-induced oxidative stress and apoptosis through the upregulation of the HO-1 and Nrf2 antioxidant genes. Food Chem Toxicol 2012; 50: 1245-1255.

12. Chung YC, Lu LC, Tsai MH, Chen YJ, Chen YY, Yao SP, Hsu CP. The inhibitory effect of ellagic acid on cell growth of ovarian carcinoma cells. Evid Based Complement Alternat Med 2013; 306705.

13. Garber JC, Barbee RW, Bielitzki JT, Clayton LA, Donovan JC, Hendriksen CFM, Kohn DF, Lipman NS, Locke PA, Melcher J, et al. Guide for the care and use of laboratory animals, ed 8. National Academic Press, 2011.

14. Shen $H$, Hu X, Liu C, Wang S, Zhang W, Gao H, Stetler $R A$, Gao $Y$, Chen J. Ethyl pyruvate protects against hypoxic-ischemic brain injury via anti-cell death and antiinflammatory mechanisms. Neurobiol Dis 2010; 37: 711 722.

15. Williams JJ, Mayurasakorn K, Vannucci SJ, Mastropietro C, Bazan NG, Ten VS, Deckelbaum RJ. N-3 fatty acid rich triglyceride emulsions are neuroprotective after cerebral hypoxic-ischemic injury in neonatal mice. PLOS One 2013; 8: e56233.

16. Keddy PGW, Dunlop K, Warford J, Samson ML, Jones QRD, Rupasingh HP, Robertson GS. Neuroprotective and anti-inflammatory effects of the flavonoid-enriched fraction AF4 in a mouse model of hypoxic-ischemic brain injury. PLoS One 2012; 7: e51324.

17. Li Y, Liang G, Wang S, Meng Q, Wang Q, Wei H. Effect of fetal exposure to isoflurane on postnatal memory and learning in rats. Neuropharmacol 2007; 53: 942-950.

18. Wang $Q$, Wang $F$, Li $X$, Yang $Q$, Li X, Xu N, Huang $Y$, Zhang $Q$, Gou $X$, Chen S. Electroacupuncture pretreatment attenuates cerebral ischemic injury through $\alpha 7$ nicotinic acetylcholine receptor-mediated inhibition of high-mobility group box 1 release in rats. $J$ Neuroinflam 2012; 9: 24.

19. Chang YC, Huang CC. Perinatal brain injury and regulation of transcription. Curr Opin Neurol 2006; 19: 141-147.

20. Blomgren $K$, Hagberg H. Free radicals, mitochondria, and hypoxia-ischemia in the developing brain. Free Radic Biol Med 2006; 40: 388-397.
21. Cheng $Y$, Deshmukh M, D'Costa A, Demaro JA, Gidday $J M$, Shah $A$, Sun $Y$, Jacquin $M F$, Johnson $E M$, Holtzman DM. Caspase inhibitor affords neuroprotection with delayed administration in a rat model of neonatal hypoxic-ischemic brain injury. J Clin Invest 1998; 101: 1992-1999.

22. Yin W, Signore AP, Iwai $M$, Cao $G$, Gao $Y$, Johnnides MJ, Hickey RW, Chen J. Preconditioning suppresses inflammation in neonatal hypoxic ischemia via Akt activation. Stroke 2007; 38: 1017-1024.

23. Zhu C, Wang X, Huang Z, Qiu L, Xu F, Vahsen N, Nilsson M, Eriksson PS, Hagberg H, Culmsee C, et al. Apoptosis-inducing factor is a major contributor to neuronal loss induced by neonatal cerebral hypoxiaischemia. Cell Death Differ 2007; 14: 775-784.

24. Han BH, D'Costa A, Back SA, Parsadanian M, Patel S, Shah AR, Gidday JM, Srinivasan A, Deshmukh M, Holtzman DM. BDNF blocks caspase-3 activation in neonatal hypoxia-ischemia. Neurobiol Dis 2000; 7: 3853.

25. Blomgren K, Zhu C, Wang X, Karlsson JO, Leverin AL, Bahr BA, Mallard C, Hagberg $H$. Synergistic activation of caspase-3 by m-calpain after neonatal hypoxiaischemia: a mechanism of "pathological apoptosis"? J Biol Chem 2001; 276: 10191-10198.

26. Hedtjarn M, Leverin AL, Eriksson K, Blomgren K, Mallard $C$, Hagberg $H$. Interleukin-18 involvement in hypoxicischemic brain injury. J Neurosci 2002; 22: 5910-5919.

27. Yao L, Kan EM, Lu J, Hao A, Dheen ST, Kaur C, Ling EA. Toll-like receptor 4 mediates microglial activation and production of inflammatory mediators in neonatal rat brain following hypoxia: role of TLR4 in hypoxic microglia. J Neuroinflam 2013; 10: 23-44

28. Jiang M, Li J, Peng Q, LiU Y, LiU W, Luo C, Peng J, Li J, Yung KK, Mo Z. Neuroprotective effects of bilobalide on cerebral ischemia and reperfusion injury are associated with inhibition of pro-inflammatory mediator production and down-regulation of JNK1/2 and p38MAPKactivation. J Neurounflam 2014; 26: 167-194

29. Irving $E A$, Bamford M. Role of mitogen- and stressactivated kinases in ischemic injury. J Cereb Blood Flow Metab 2002; 22: 631-647.

30. Kovalska M, Kovalska L, Pavlikova M, Janickova $M$, Mikuskova K, Adamkov M, Kaplan P, Tatarkova Z, Lehotsky J. Intracellular signalling MAPK pathway after cerebral ischemia-reperfusion injury. Neurochem Res 2012; 37: 1568-1577.

31. Li Q, Verma IM. NF-kappaB regulation in the immune system. Nat Rev Immunol 2002; 2: 725-734.

32. Pomerantz JL, Baltimore $D$. Two pathways to NFkappaB. Mol Cell 2002; 10: 693-695.

33. Murugan M, Sivakumar V, Lu J, Ling EA, Kaur $C$. Expression of $\mathrm{N}$-methyl daspartate receptor subunits in amoeboid microglia mediates production of nitric oxide via NF-kappaB signaling pathway and oligodendrocyte cell death in hypoxic postnatal rats. Glia 2011; 59: 521 539 
Chen et al

34. Glass CK, Saijo K, Winner B, Marchetto MC, Gage FH. Mechanisms underlying inflammation neurodegeneration. Cell 2010; 140: 918-934. 